\title{
The establishment and Simulation of the dynamic model of the Four rotor aircraft \\ Wu Wei ${ }^{1, a}$, Kai Fan Zhao ${ }^{2, b}$ and Qiang Wang ${ }^{2, c}$ \\ Wuhan university of technology, Wuhan 430063, China;

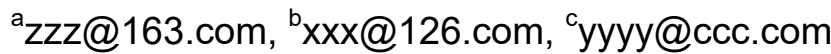

Keywords: Four rotor aircraft, Dynamic model, matlab Simulink, Simulation analysis.

\begin{abstract}
With the development of space technology and mature, four rotor aircraft with its low cost, high performance, unique structure and flight mode and other characteristics, are widely used in military and civil fields. According to the basic characteristics of four rotor aircraft prototype and flight characteristics, build up the dynamic and power system dynamics model. First the assumptions of the model, and analysis of the stress, dynamic model of the four rotor aircraft by the laws of physics. The simulation model was established in the MATLAB Simulink environment. The analysis of the simulation results, the model is verified the feasibility and validity of the model can be obtained, through the design of the control method, for the future spacecraft attitude solution to lay a good foundation work. This paper proposes a method of establishing a four rotor aircraft model, provides the reference for the further study of the four rotor flight system.
\end{abstract}

\section{Introduction}

Four rotor aircraft have the Capable of hovering and vertical takeoff and landing.Its structure is simple,compact and flexible.these advantages determine its wide range of applications ${ }^{[1]}$. With applications more and more, the four rotor aircraft control requirements are increasingly high.However, the system has a significant nonlinear, less driving characteristic, which makes it difficult to control ${ }^{[2]}$. In view of the problem of aircraft control, it is necessary to get a mathematical model and then study the predictive control method by force analysis of the aircraft body. Matlab software has a Simulink emulator to achieve a good mathematical model analysis and design ${ }^{[3]}$. The mathematical model of the four rotor aircraft is very complex. So it is difficult to accurately model the model into a computer and do further analysis and simulation. But Simulink is a good solution to this problem. In this paper, we analyze the dynamic model of aircraft based on Simulink.

The coordinate system used in this model is Inertial coordinate system $i$ and Body coordinate system $b$. Because the four rotor UAV flight height is low and the battery life is short. Therefore, the influence of the earth rotation, the gravity acceleration, height and the latitude changes is not considered. The $\mathrm{X}$ axis of the inertial system refers to the East, the $\mathrm{Y}$ axis refers to the north, the $\mathrm{Z}$ axis is perpendicular to the xoy plane (far from the inner earth).

The general structure of the four rotor UAV is the cross configuration of the four propeller. Each propeller provides the center of the body with a certain lift and torque. But the four propeller are not exactly the same. Since the four rotor UAV four propeller is paired.Therefore, the opposite of the propeller turn to the contrary, so that two pair of the propeller to provide lift to the aircraft. Moreover, the total torque generated by the four propeller is zero ${ }^{[4]}$. 


\section{Model hypothesis}

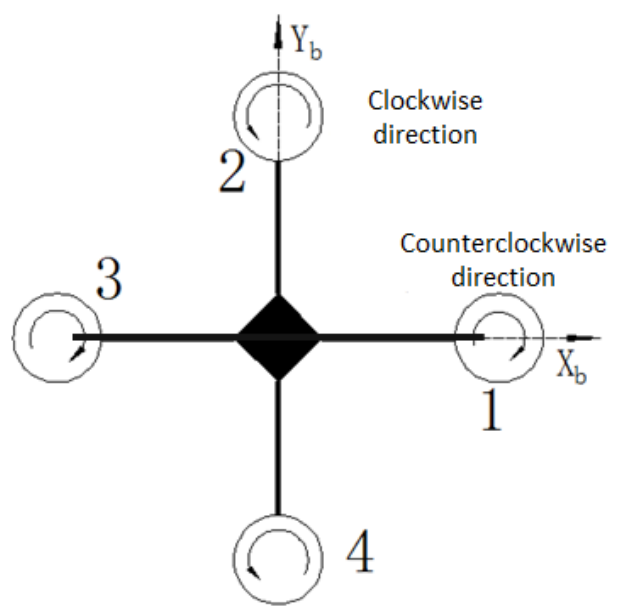

Figure 1 the structure diagram of four rotor

As shown in Figure 1, the model of the four rotor UAV is assumed as follows:

Airframe: Rectangular body whose quality uniform distribution and center is at the origin. The upper and lower two surfaces are square. The length of the side for it is $a_{b}$.it's high is $h_{b}$.2Paddle:It is a thin circular plate,the Mass of which distribution in the plane of the body coordinate system.Its Radius is $l_{r}$, its quality is $m_{r}$.3Electric motor: It's a cylinder the mass of which is uniformly distributed and the center of its gravity is in the body coordinate system $x^{b} o y^{b}$. The radius is $r_{m}$, the height is $h_{m}$, the quality is $m_{m}$.4The mass of the connecting member is calculated in the body weight. The connecting member arm length is $r$ (Distance from the center of the motor to the origin).Motor 1and 3 turn clockwise and motor 2 and 4 turns counterclockwise. 6 UAV is seen as a rigid body.7 Without considering the characteristics of the propeller waving.8 The air resistance is proportional to the speed of flight, and the direction is opposite to the speed and the action point is located in the center of the UAV.9 The rotational air resistance torque in flight is proportional to the angular velocity and the direction is opposite to the angular velocity.

Under the Airframe coordinate:

Motor 1,2,3,4 position coordinates are:

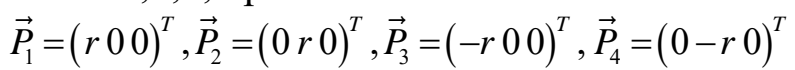

The Inertia tensor of the origin is calculated as follows:

Airframe:

$$
I_{b}^{b}=\left[\begin{array}{ccc}
\frac{1}{12} m_{b}\left(a^{2}+h^{2}\right) & 0 & 0 \\
0 & \frac{1}{12} m_{b}\left(a^{2}+h^{2}\right) & 0 \\
0 & 0 & \frac{1}{6} m_{b} a^{2}
\end{array}\right]
$$

Propeller:

$$
I_{r 1}^{b}=I_{r 3}^{b}=\left[\begin{array}{ccc}
\frac{1}{4} m_{r} l^{2} & 0 & 0 \\
0 & \frac{1}{4} m_{r} l^{2}+m_{r} r^{2} & 0 \\
0 & 0 & \frac{1}{2} m_{r} l^{2}+m_{r} r^{2}
\end{array}\right] I_{r 2}^{b}=I_{r 4}^{b}=\left[\begin{array}{ccc}
\frac{1}{4} m_{r} l^{2}+m_{r} r^{2} & 0 & 0 \\
0 & \frac{1}{4} m_{r} l^{2} & 0 \\
0 & 0 & \frac{1}{2} m_{r} l^{2}+m_{r} r^{2}
\end{array}\right]
$$

Motor: 


$$
\begin{aligned}
& I_{m}^{b}=\sum_{i=1}^{4} I_{m i}^{b}=\left[\begin{array}{ccc}
\frac{1}{3} m_{m}\left(3 r_{m}^{2}+h_{m}^{2}+6 r^{2}\right) & 0 & 0 \\
0 & \frac{1}{3} m_{m}\left(3 r_{m}^{2}+h_{m}^{2}+6 r^{2}\right) & 0 \\
0 & 0 & 2 m_{m}\left(r_{m}^{2}+2 r^{2}\right)
\end{array}\right] I_{m 1}^{b}=I_{m 3}^{b}=\left[\begin{array}{ccc}
\frac{1}{12} m_{m}\left(3 r_{m}^{2}+h_{m}^{2}\right) & 0 & 0 \\
0 & \frac{1}{12} m_{m}\left(3 r_{m}^{2}+h_{m}^{2}\right)+m_{m} r^{2} & 0 \\
0 & 0 & \frac{1}{2} m_{m} I^{2}+m_{m} r^{2}
\end{array}\right] \\
& I_{m 2}^{b}=I_{m 4}^{b}=\left[\begin{array}{ccc}
\frac{1}{12} m_{m}\left(3 r_{m}^{2}+h_{m}^{2}\right)+m_{m} r^{2} & 0 & 0 \\
0 & \frac{1}{12} m_{m}\left(3 r_{m}^{2}+h_{m}^{2}\right) & 0 \\
0 & 0 & \frac{1}{2} m_{m} I^{2}+m_{m} r^{2}
\end{array}\right]
\end{aligned}
$$

Total inertia tensor: $I^{b}=I_{b}^{b}+I_{r}^{b}+I_{m}^{b}$.

\section{UAV force analysis}

In order to establish a dynamic model of four rotor aircraft, it is necessary to establish the environment coordinate system,so that the various physical quantities can be reflected. In fact, the physical volume obtained by inertial navigation sensors determine four rotor position and attitude. Among them, in order to facilitate the description of rotating and moving aircraft, the body coordinate system; to determine the four rotor aircraft attitude, position, should adopt the ground coordinate system. The ground coordinate system is a coordinate system fixed with the earth. The ox axis is an arbitrary direction pointing to the ground plane.Ox axis vertical plane, oy and oz constitute the vertical axis, which coordinate.Ignore the curvilinear motion of earth rotation and centroid, the coordinate system can be regarded as the inertial coordinate system. The aircraft position and attitude, velocity and angular velocity are relative to the coordinate system to measure. The 3D orthogonal cartesian coordinate system fixed on the aircraft is called the aircraft body coordinate system, whose origin is located at the center of gravity of the aircraft.Ox is located in the aircraft reference plane parallel to the axis of the body and pointing to the front of the aircraft, the oy vertical axis in aircraft reference plane and point on the right side of the aircraft, the oz axis in reference plane vertical to the xoy plane, pointing to the top of the aircraft.

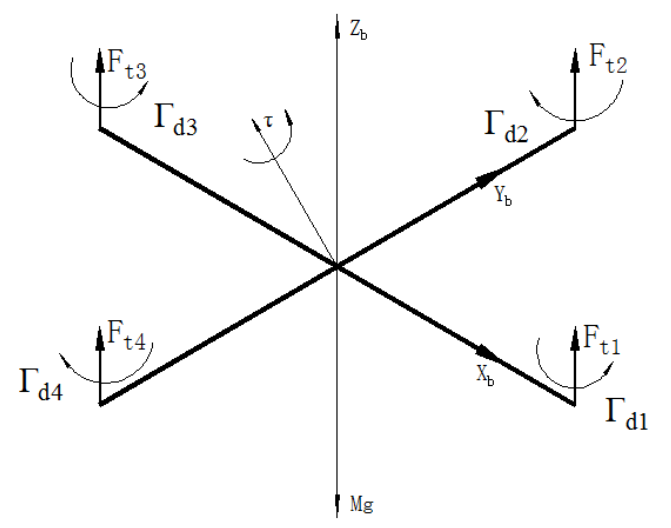

Figure 2 the UAV force analysis

In Figure 2, the UAV is effected by gravity $M g$,translational rotational air resistance $f$, air resistance $\tau$, propeller thrust $F$,propeller resistance moment $\Gamma_{d}$.

Gravity: $M g=\left[m_{b}+4 \times\left(m_{m}+m_{r}\right)\right] g$,the direction along the negative direction of $z^{i}$;Translational rotational air resistance: $f=\gamma_{t} v^{b} \gamma_{t}$ translational air drag coefficient, $v^{b}$ the UAV in the body coordinate speed; Turn the air resistance moment: $\tau=\gamma_{\mathrm{r}} \Omega^{b} \gamma_{\mathrm{r}}$ turn the air drag coefficient, $\Omega^{b}$ the UAV angular velocity in body coordinates, the direction is opposite to the direction $\Omega^{b}$. Propeller thrust: $F_{t}=F_{t 1}+F_{t 2}+F_{t 3}+F_{t 4}, F_{t i}=k \omega_{i}^{2} i=1,2,3,4, k$ is the propeller of lift coefficient,the direction along the positive direction of axis $z^{b}$; Propeller resistance moment: 
$\Gamma=\Gamma_{d 1}-\Gamma_{d 2}+\Gamma_{d 3}-\Gamma_{d 4}, \Gamma_{d i}=\alpha \omega_{i}^{2} i=1,2,3,4, \alpha$ is the propeller resistance coefficient,its direction is opposite to the direction of the angular velocity of propeller.

\section{UAV dynamic model}

To establish the dynamics model of UAV uses Euler angle or four element mathematical tools generally.Euler angle is used to determine the position of the rigid body rotates around a fixed point and a group of 3 independent angle parameters, composed of yaw, pitch angle and roll angle. According to the different rotation order, Euler angle can have 12 kinds of combination. Each combination has different meaning. We define the Euler angle rotation order:the first rotating around the $z^{i}$ rotary $\psi$; and then around the new $y^{i}(1)$ rotary $\theta$; finally around the new $x^{i}(1)$ rotary $\phi$. Then we get the direction cosine matrix DCM as follows:

$$
R=\left(R_{z y x}(\psi, \theta, \phi)\right)^{T}=\left[\begin{array}{ccc}
c_{\theta} c_{\psi} & s_{\phi} s_{\theta} c_{\psi}-c_{\phi} s_{\psi} & c_{\phi} s_{\theta} c_{\psi}+s_{\phi} s_{\psi} \\
c_{\theta} s_{\psi} & s_{\phi} s_{\theta} s_{\psi}+c_{\phi} c_{\psi} & c_{\phi} s_{\theta} s_{\psi}-s_{\phi} c_{\psi} \\
-s_{\theta} & s_{\phi} c_{\theta} & c_{\phi} c_{\theta}
\end{array}\right]
$$

But when $\cos \theta=0$, there will be a gimbal lock phenomenon, Euler angle calculation failure. Because when $\theta= \pm \pi / 2$ euler angle is singular and lead to failure of attitude solution. In order to avoid the singularity of Euler angles, with four element mathematical instead of Euler angles to describe the rigid body rotation.

To establish the dynamic equation, firstly various force aircraft are represented by vector matrix:gravity,translational rotational air resistance.air resistance,propeller thrust,propeller resistance moment. In the ground coordinate system, according to Newton's second law:

$$
M \overrightarrow{\vec{v}^{\prime}}=q \circ\left(\begin{array}{c}
0 \\
0 \\
k \sum_{i=1}^{4} \omega_{i}^{2}
\end{array}\right) \circ q^{-1}+\left(\begin{array}{c}
0 \\
0 \\
-M g
\end{array}\right)-q \circ\left(\gamma_{t}\left(q^{-1} \circ \vec{v}^{i} \circ q\right)\right) \circ q^{-1}, \vec{v}^{b}=\left(q^{-1} \circ \vec{v}^{i} \circ q\right)
$$

$\mathrm{o}$ is four element mathematical multiplication. And because the angular momentum conservation, according to the nature of the direction cosine:

$$
\left\{\begin{array}{l}
I^{i}=R \cdot I^{b} \cdot R^{-1} \\
I_{r}^{i}=R \cdot I_{r}^{b} \cdot R^{-1} \\
\mathrm{r}^{i}=R \cdot \stackrel{\mathrm{r}}{ }^{b} \\
\Omega^{i}=R \cdot \stackrel{\mathrm{r}}{\Gamma}^{b} \\
\mathrm{r}^{i}=R \cdot \Gamma^{b}
\end{array}\right.
$$

Getting the momentum of UAV:

$$
L=R \cdot I^{b} \cdot R^{-1} \cdot R \cdot \stackrel{\mathrm{r}}{\Omega^{b}}+\sum_{j=1}^{4} R \cdot I_{r j}^{b} \cdot R^{-1} \cdot R \cdot \stackrel{\mathrm{r}_{\omega}^{b}}{\omega_{j}}=R \cdot I^{b} \cdot \stackrel{\mathrm{r}^{b}}{\Omega^{b}}+\sum_{j=1}^{4} R \cdot I_{r j}^{b} \cdot \stackrel{\mathrm{r}}{\omega}_{j}
$$

Then the stress analysis of rotor and propeller, getting the size of the electromagnetic torque:

$$
\mathbb{R}_{m i}^{\&}=-\frac{R_{e s} \Gamma_{m i}}{L}-\frac{K_{\varphi}^{2}}{L} \omega_{i}+\frac{K_{\varphi}}{L} u_{i}
$$

$\Gamma_{m i}$ :Electromagnetic torque; ${ }^{\Gamma_{d i}}$ : Propeller resistance moment; ${ }^{\tau_{i}}$ :friction torque of the motor shaft; $u$ : Motor voltage;L: Inductance coefficient; ${ }^{K}{ }_{\varphi}$ : EMF coefficient; ${ }^{R}{ }_{e s}$ : Motor resistance;

The rotor and propeller shaft using angular momentum conservation theorem:

angular velocity: $\&_{i}=\frac{K_{\varphi} i}{J}-\frac{\alpha \omega_{i}^{2}}{J}-\frac{f_{m} \omega_{i}}{J}$ 
Laplace transform of the voltage $u=R_{e s} i+L \frac{d i}{d t}+K_{\varphi} \omega$ and the angular velocity $\dot{\omega}_{i}=\frac{K_{\varphi} i}{J}-\frac{\alpha \omega_{i}^{2}}{J}-\frac{f_{m} \omega_{i}}{J}$, linear at the $\omega_{0}$, obtaining: $w 0=35.5$.

So:

$$
H(s)=\frac{7933}{s^{2}+178 s+1947} \approx \frac{7933}{(s+11.9)(s+166.1)}
$$

This UAV model is established, all the formulas are summarized as follows:

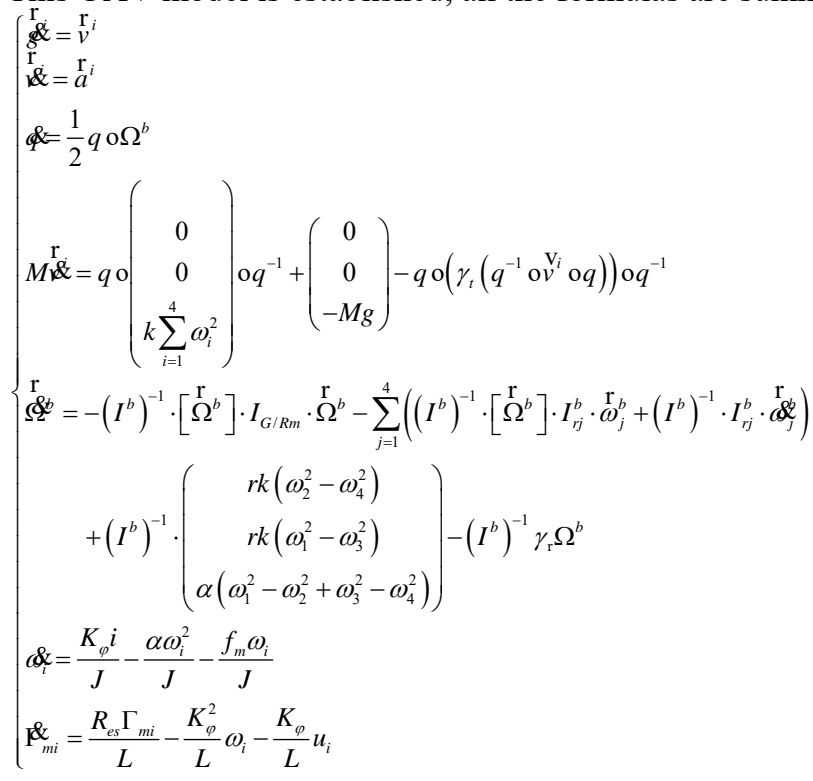

\section{Dynamic model checking}

In order to verify the correctness of the dynamic model,establishing two models: one is the four element model; two is the Euler angle model ${ }^{[5]}$.As Figure, the same input voltage of the two models, compare the output attitude difference. As figure 3 block diagram of model checking, four motor voltage are respectively $9 \mathrm{~V}, 9.1 \mathrm{~V}, 8.9 \mathrm{~V}, 9.2 \mathrm{~V}$. The Simulink simulator calculation error to eps (10-14) . The simulation results shown in Figure 4, figure 5.The step below Figure 4 is due to Euler angle leads to the gimbal lock. So it can only be compared before the results of $10 \mathrm{~s}$. As you can see from the results, the angular velocity and velocity error magnitude is $10-15$. It can be concluded that the two models are basically the same, Then verify the correctness of the model.

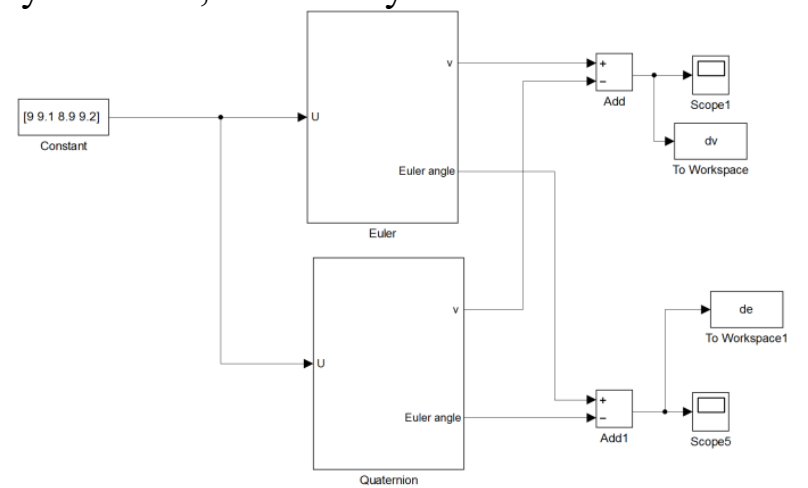

Figure 3 Model test diagram 

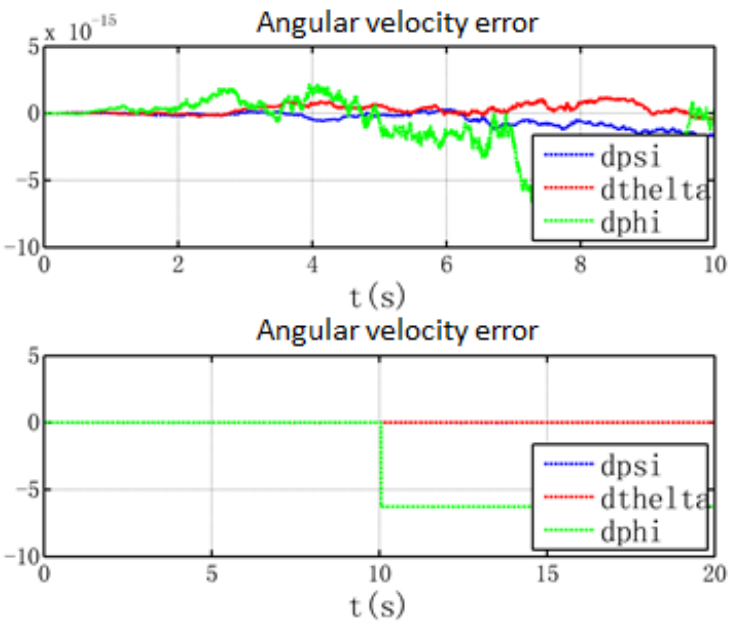

Figure 4 Angular velocity error

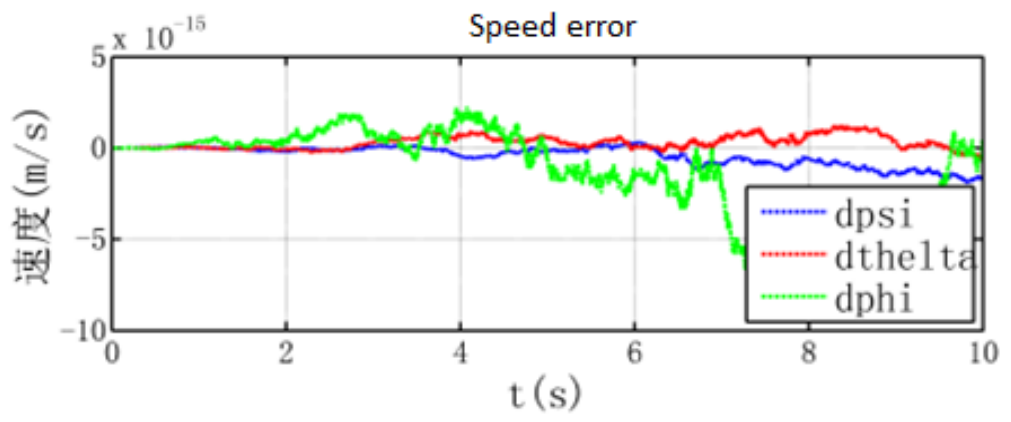

Figure 5 Speed error

\section{Summary}

The four rotor aircraft as the research object, the parameters of the first set of four rotor physical model and the external environment of physical quantity, assumptions of the model. On this basis, we establishes the kinematics model of force analysis. With four element number and Euler angle two mathematical tools in Simulink simulator simulation, comparison of the obtained results deviation, found that the results are consistent, indicating the validity of the model.It provides a reference for the further study of the motion characteristics of four rotor aircraft to design method of control of four rotor aircraft based on this model.

\section{References}

[1] Pan QingPei, Four rotor aircraft design and stability control research[D], China university of science and technology, 2011.

[2] Su JingYa,Zhang Ruifeng, Cai KaiYuan. Based on four rotor aircraft noise differentiator control [J]. Control theory and application of preceding 2009:827-832.

[3] Hu Qiyi, Four rotor aircraft attitude estimation and optimization control research [D]. Hangzhou university of electronic science and technology, 2014.

[4] NieBoWen, Small four rotor unmanned helicopter modeling and control method research [D]. National university of defense technology, 2006.

[5] Wang YongLin,Matlab/Simulink environment whole process unmanned aerial vehicle (uav) flight simulation technology research [D]. Nanjing university of aeronautics and astronautics, 2006. 